\title{
On semigroups admitting ring structure
}

\author{
Ryszard Mazurek
}

Dedicated to Professor Jan Skowroński.

Received: 12 October 2010 / Accepted: 5 June 2011 / Published online: 17 June 2011

(C) The Author(s) 2011. This article is published with open access at Springerlink.com

\begin{abstract}
A right-chain semigroup is a semigroup whose right ideals are totally ordered by set inclusion. The main result of this paper says that if $S$ is a right-chain semigroup admitting a ring structure, then either $S$ is a null semigroup with two elements or $s S=S$ for some $s \in S$. Using this we give an elementary proof of Oman's characterization of semigroups admitting a ring structure whose subsemigroups (containing zero) form a chain. We also apply this result, along with two other results proved in this paper, to show that no nontrivial multiplicative bounded interval semigroup on the real line $\mathbb{R}$ admits a ring structure, obtaining the main results of Kemprasit et al. (ScienceAsia 36: 85-88, 2010).
\end{abstract}

Keywords Semigroup admitting a ring structure · Right-chain semigroup · Multiplicative interval semigroup

\section{Introduction}

If $(R,+, \cdot)$ is a ring, then the multiplicative structure $(R, \cdot)$ of the ring is by definition a semigroup with zero. On the other hand, as it is shown by examples of left zero semigroups (i.e. semigroups $S$ such that $x y=x$ for any $x, y \in S$ ) with zero adjoined, not every semigroup $(S, \cdot)$ with zero admits a ring structure, that is an addition + cannot always be defined on $S$ such that $(S,+, \cdot)$ is a ring.

The problem of characterizing semigroups admitting ring structure was studied by many authors (see [9] for a brief survey of results on the topic, obtained till 1970). As proved by S.R. Kogalovski [5], it is impossible to characterize the class of all

Communicated by Michael W. Mislove.

R. Mazurek (凶)

Faculty of Computer Science, Bialystok University of Technology, Wiejska 45A, 15-351 Białystok, Poland

e-mail: r.mazurek@pb.edu.pl 
semigroups admitting ring structure axiomatically, and thus to obtain worthwhile results the problem is often restricted to particular classes of semigroups. Sometimes it makes the problem quite easy. For example, since an abelian group structure can be defined on every nonempty set (interestingly, the statement is equivalent to the Axiom of Choice, see [3]), every null semigroup (i.e. a semigroup $S$ such that $x y=0$ for any $x, y \in S$ ) admits a ring structure. In general, however, the problem of identifying semigroups admitting ring structure within a given class of semigroups seems to be difficult.

In this paper all semigroups as well as all subsemigroups are with zero 0 , semigroup operation is written multiplicatively, and all rings are associative but not necessarily with unity. If $S$ is a semigroup such that an addition + may be defined on $S$ so that $(S,+, \cdot)$ is a ring, then we say that $S$ admits a ring structure. Such a semigroup $S$ will also be called a ring semigroup.

In Sect. 1 of this paper we study right-chain semigroups admitting ring structure. Recall that a semigroup $S$ is said to be a right-chain semigroup if the right ideals of $S$ are totally ordered by set inclusion [2]. The main result of the paper is Theorem 1.1 which says that if $S$ is a right-chain semigroup admitting a ring structure, then either $S$ is a null semigroup with two elements or $s S=S$ for some $s \in S$. Using this result, in Sect. 2 we give an elementary proof of G. Oman's characterization [7, Theorem 1] of semigroups admitting a ring structure whose subsemigroups form a chain. In Sect. 3 we apply Theorem 1.1, along with Theorems 1.3 and 3.1, to give alternative and shorter proofs of main results of Y. Kemprasit et al. from [4] which imply that the trivial semigroups $\{0\}$ and $\{1\}$ are the only multiplicative bounded interval semigroups on the real line $\mathbb{R}$ admitting a ring structure.

If $S$ is a semigroup and $a \in S$, then the right ideal of $S$ generated by $a$ is denoted by $a S^{1}$, i.e. $a S^{1}=\{a\} \cup a S=\{a\} \cup\{a s \mid s \in S\}$, and the subsemigroup of $S$ generated by $a$ is denoted by $\langle 0, a\rangle$, i.e. $\langle 0, a\rangle=\{0\} \cup\left\{a^{n}: n \in \mathbb{N}\right\}$. If $S$ is a semigroup with unity 1 , then we assume that $1 \neq 0$. For a set $A$, the cardinality of $A$ is denoted by Card $A$.

\section{On right-chain semigroups admitting ring structure}

A right-chain semigroup is a semigroup whose right ideals are totally ordered by set inclusion [2]. It is easy to see that a semigroup $S$ is a right-chain semigroup if and only if $a S^{1} \subseteq b S^{1}$ or $b S^{1} \subseteq a S^{1}$ for any $a, b \in S$.

Theorem 1.1 Let $S$ be a right-chain semigroup such that $S \neq S$ for any $s \in S$. Then $S$ is a ring semigroup if and only if $S$ is a null semigroup with two elements.

Proof The "if" part is clear. For the "only if" part, assume $(S,+, \cdot)$ is a ring for some addition + . We first prove that $S$ is a null semigroup. For contradiction, suppose $x y \neq$ 0 for some $x, y \in S$. Since $S$ is a right-chain semigroup, we have $x S^{1} \subseteq(x+x y) S^{1}$ or $(x+x y) S^{1} \subseteq x S^{1}$, and since $x \neq x+x y$, in both cases it follows that $x \in x S$. Hence $x=x s$ for some $s \in S$. Set $I=\{t-s t \mid t \in S\}$. If $s \in I$, then $s=t-s t$ for some $t \in S$, which leads to $x=x s=x(t-s t)=x t-(x s) t=x t-x t=0$, a contradiction. Thus 
$s \notin I$, and since $I$ is a right ideal of the right-chain semigroup $S, I \subseteq s S$ follows. Hence $t-s t \in s S$ for any $t \in S$, which implies $S=s S$, a contradiction. Thus $S$ is a null semigroup.

To complete the proof, we show that $S$ is a semigroup with two elements. If $S=$ $\{0\}$, then $s S=S$ for $s=0$, a contradiction. Thus $S$ contains a nonzero element. Suppose $S$ contains two different nonzero elements $a, b$. Since $S$ is a null semigroup, $a S^{1}=\{0, a\}$ and $b S^{1}=\{0, b\}$ are incomparable right ideals of $S$, contradicting that $S$ is a right-chain semigroup. Hence $\operatorname{Card} S=2$.

Obvious examples of right-chain semigroups are semigroups $S$ such that $S=$ $\langle 0, s\rangle$ for some $s \in S$; for the purpose of this paper, let us call such a semigroup $S$ a 0 -cyclic semigroup. Since the multiplicative group of a finite field $(F,+, \cdot)$ is cyclic, $(F, \cdot)$ is a 0 -cyclic ring semigroup. Below we give a new proof of Corollary 3.3(3) of [1] which says that up to isomorphism, with exception of the trivial semigroup and the null semigroup with two elements, 0 -cyclic ring semigroups are exactly multiplicative semigroups of finite fields, i.e. multiplicative semigroups of the form $\mathbb{F}_{p^{n}}$, where $p$ is a prime and $n \in \mathbb{N}$. As an immediate consequence of the corollary we obtain a well-known result that all 0-cyclic ring semigroups are finite (e.g. see [10, Corollary 1.4]).

Corollary 1.2 A semigroup $S$ is a 0 -cyclic ring semigroup if and only if either $S=$ $\{0\}$, or $S=\{0, s\}$ with $s \neq 0$ and $s^{2}=0$, or $S \cong \mathbb{F}_{p^{n}}$ for some prime $p$ and $n \in \mathbb{N}$. In particular, every 0 -cyclic ring semigroup is finite.

Proof Assume $S$ is a nonzero 0-cyclic ring semigroup, i.e. $S=\{0\} \cup\left\{s^{n}: n \in \mathbb{N}\right\}$ for some $s \in S \backslash\{0\}$. By Theorem 1.1, it suffices to consider the case where $s^{k} S=S$ for some $k \in \mathbb{N}$. Then $s=s^{k} x$ for some $x \in S$, which implies $s=s^{m}$ for some $m \geq 2$. Thus $S$ is finite, $1=s^{m-1}$ is an identity element of $S$, and each nonzero element of $S$ is invertible. Since $S$ is a commutative ring semigroup, it follows that $S$ is isomorphic to a multiplicative semigroup of a finite field.

Obviously, the semigroups $\{0\},\{0, s\}$ with $s^{2}=0$, and $\mathbb{F}_{p^{n}}$ are 0 -cyclic ring semigroups.

Condition (1.1) of our next result with $A=J$ obviously is satisfied for any rightchain semigroup. We state the result in a more general setting for further applications in Sect. 3.

Theorem 1.3 Let $S$ be a semigroup such that $e S=S$ for some $e \in S$, and let $J=\{s \in$ $S: s S \neq S\}$. If $S$ is a ring semigroup, then for any subset $A$ of $S$ with the following property:

$$
\text { for any } x, y \in A \text { there exists } z \in J \text { with } x S \subseteq z S \text { and } y S \subseteq z S \text {, }
$$

we have $\operatorname{Card} A \leq \operatorname{Card}(S \backslash A)$ and $\operatorname{Card} S \leq 2 \cdot \operatorname{Card}(S \backslash A)$. 
Proof Let + be an addition such that $(S,+, \cdot)$ is a ring and let $x \in A$. If $e+x \in A$, then for some $z \in J$ we have $(e+x) S \subseteq z S$ and $x S \subseteq z S$, which leads to

$$
S=e S=((e+x)-x) S \subseteq(e+x) S+x S \subseteq z S+z S \subseteq z S \neq S,
$$

a contradiction. Hence for any $x \in A$ we have $e+x \in S \backslash A$, and thus by setting $\varphi(x)=e+x$ we obtain an injection of $A$ into $S \backslash A$, which proves that $\operatorname{Card} A \leq$ $\operatorname{Card}(S \backslash A)$. Therefore, $\operatorname{Card} S=\operatorname{Card} A+\operatorname{Card}(S \backslash A) \leq 2 \cdot \operatorname{Card}(S \backslash A)$.

As a consequence of Theorems 1.1 and 1.3, we obtain the following result of L.J.M. Lawson [6] (see [9, Theorem 4.7]).

Corollary 1.4 Let $S$ be a right-chain semigroup such that for any $x, y \in S, x S^{1}=$ $y S^{1}$ implies $x=y$. If $S$ is a ring semigroup, then $\operatorname{Card} S \leq 2$.

Proof Set $J=\{s \in S: s S \neq S\}$. If $S=J$, then the result follows from Theorem 1.1. We are left with the case where $S \neq J$. If $x, y \in S \backslash J$, then $x S^{1}=S=y S^{1}$, and by hypothesis $x=y$ follows. Hence in this case $S \backslash J$ consists of only one element and the result follows from Theorem 1.3 by taking $A=J$.

\section{Ring semigroups whose subsemigroups form a chain}

In [7] G. Oman characterized ring semigroups whose subsemigroups form a chain with respect to set inclusion (see Theorem 2.1 below). His proof however uses other nontrivial results, in particular Mihăilescu's Theorem on Catalan's Conjecture (see [7] for details). We note that semigroups whose subsemigroups form a chain are right-chain semigroups and thus Theorem 1.1 applies to this class of semigroups. Below we present an elementary and selfcontained proof of Oman's result.

Theorem 2.1 ([7, Theorem 1]) A semigroup $S$ is a ring semigroup whose subsemigroups are totally ordered if and only if one of the following holds:

(1) $S=\{0\}$,

(2) $S$ is a null semigroup with two elements,

(3) $S \cong \mathbb{F}_{p}$, where $p$ is a prime and $p=2^{n}+1$ for some $n \in \mathbb{N} \cup\{0\}$,

(4) $S \cong \mathbb{F}_{2^{n}}$, where $n \in \mathbb{N}$ is such that $2^{n}-1$ is a prime,

(5) $S \cong \mathbb{F}_{9}$.

Proof It is easy to check that the semigroups in (1)-(5) satisfy the condition. Conversely, suppose $S$ is a ring semigroup whose subsemigroups form a chain. If $S=\{0\}$, then $S$ is of type (1). Assume that $S \neq\{0\}$. Since for any $x, y \in S \backslash\{0\}$ we have $\langle 0, x\rangle \subseteq\langle 0, y\rangle$ or $\langle 0, y\rangle \subseteq\langle 0, x\rangle, x=y^{n}$ or $y=x^{n}$ for some $n \in \mathbb{N}$, which clearly implies $x y=y x$. Thus $S$ is commutative. If $s S \neq S$ for any $s \in S$, then $S$ is of type (2) by Theorem 1.1. Thus we assume that $s=S$ for some $s \in S$ and $S \neq\{0\}$. Then $s=s e$ for some $e \in S \backslash\{0\}$. Since for any $x \in S=s S$ there exists $y \in S$ with $x=s y$, we have $e x=e s y=s e y=s y=x$ and thus $e$ is an identity element of $S$ and $\{0, e\}$ 
is a subsemigroup of $S$. Since subsemigroups of $S$ form a chain, for any $t \in S \backslash\{0\}$ we have $\{0, e\} \subseteq\langle 0, t\rangle$ and thus $t^{n}=e$ for some $n \in \mathbb{N}$, which shows that $S \backslash\{0\}$ is a torsion abelian group.

Let + be an addition such that $(S,+, \cdot)$ is a ring. From the preceding it follows that $(S,+, \cdot)$ is a field of prime characteristic $p$. We claim that

any finite subfield $F$ of $(S,+, \cdot)$ belongs to family (3), (4) or (5),

that is, either $F$ is isomorphic to a field $\mathbb{F}_{p}$ with $p$ as described in (3), or $F$ is isomorphic to a field $\mathbb{F}_{2^{n}}$ with $n$ as described in (4), or $F \cong \mathbb{F}_{9}$. Once we have proved this, it will follow that $S$ is finite, and thus by (2.1) $S$ itself belongs to family (3), (4) or (5). Indeed, since the multiplicative group $S \backslash\{0\}$ is torsion, every element of $S$ belongs to a finite subfield of $S$. Hence, if $S$ were infinite, then $S$ would contain infinitely many finite subfields. But, assuming (2.1), if $p \notin\{2,3\}$, then the only finite subfield of $S$ is $\mathbb{F}_{p}$, and if $p=3$, then the only possible finite subfields of $S$ are $\mathbb{F}_{3}$ and $\mathbb{F}_{9}$. We are left with the case where $p=2$. Suppose $\mathbb{F}_{2^{m_{1}}}$ and $\mathbb{F}_{2^{m_{2}}}$ are different subfields of $S$ with $2<m_{1}<m_{2}$. Since subsemigroups of $S$ form a chain, it follows that $\mathbb{F}_{2^{m_{1}}} \varsubsetneqq \mathbb{F}_{2^{m_{2}}}$. Hence, assuming (2.1), $2^{m_{1}}-1$ and $2^{m_{2}}-1$ are different primes such that $2^{m_{1}}-1$ divides $2^{m_{2}}-1$. This contradiction shows that if $p=2$, then $S$ contains no more than two finite subfields.

To prove (2.1), suppose $F$ is a finite subfield of $S$. Then $F=\mathbb{F}_{p^{m}}$ for some $m \in \mathbb{N}$ and the multiplicative group $F \backslash\{0\}$ is a cyclic subgroup of $S \backslash\{0\}$ of order $p^{m}-1$. Thus for any $a, b \in \mathbb{N}$, if $p^{m}-1=a b$, then the group $F \backslash\{0\}$ contains subgroups $A, B$ of order $a, b$ respectively, and since the subsemigroups $A \cup\{0\}$ and $B \cup\{0\}$ of $S$ are comparable, it follows that $A \subseteq B$ or $B \subseteq A$, and so $a$ divides $b$ or $b$ divides $a$. Therefore, if $p^{m}-1 \neq 1$, then

$$
p^{m}-1=q^{k} \quad \text { for some prime } q \text { and } k \in \mathbb{N} \text {. }
$$

Assume $p \neq 2$. Then $p^{m}-1 \neq 1$, hence (2.2) holds with $q=2$, i.e. $p^{m}-1=2^{k}$. If $m$ is even, then $2^{k}=\left(p^{\frac{m}{2}}-1\right)\left(p^{\frac{m}{2}}+1\right)$, hence $p^{\frac{m}{2}}-1=2^{\alpha}$ and $p^{\frac{m}{2}}+1=2^{\beta}$ for some $\alpha, \beta \in \mathbb{N}$. Thus $2=p^{\frac{m}{2}}+1-\left(p^{\frac{m}{2}}-1\right)=2^{\beta}-2^{\alpha}$, so $\alpha=1$, and thus $p=3$ and $m=2$. Hence $F \cong \mathbb{F}_{9}$ is of type (5). We are left with the case where $m$ is odd. Then $2^{k}=(p-1) \sum_{i=0}^{m-1} p^{i}$. Since $m$ and $p$ are odd, $\sum_{i=0}^{m-1} p^{i}$ is odd too, thus $\sum_{i=0}^{m-1} p^{i}=1$, and $m=1$ follows. Hence $F \cong \mathbb{F}_{p}$, where $p=2^{k}+1$ is a prime, and thus $F$ belongs to the family (3).

Now assume $p=2$. If $2^{m}-1=1$, then $m=1$ and $F \cong \mathbb{F}_{2}$ belongs to the family (3). Assume $2^{m}-1 \neq 1$. Then from (2.2) we have $2^{m}-1=q^{k}$ for some prime $q$ and $k \in \mathbb{N}$. If $k$ is even, then $q^{k} \equiv 1(\bmod 4)$, thus $2^{m} \equiv 2(\bmod 4)$. Hence $m=1$, so $q^{k}=1$, a contradiction. If $k$ is odd, then $2^{m}=(1+q) \sum_{i=0}^{k-1}(-q)^{i}$. Since $k$ and $q$ are odd, so is $\sum_{i=0}^{k-1}(-q)^{i}$, and thus $2^{m}=1+q$. Hence $F \cong \mathbb{F}_{2^{m}}$, where $m \in \mathbb{N}$ and $2^{m}-1=q$ is a prime, and thus $F$ belongs to the family (4). 


\section{Bounded interval semigroups on $\mathbb{R}$ admitting ring structure}

It follows from [8, p. 273] that the only bounded interval semigroups on the real line $\mathbb{R}$, with ordinary multiplication, are the trivial intervals $\{0\}=[0,0]$ and $\{1\}=[1,1]$, and intervals of the following types:

(i) $[0, b)$ or $[0, b]$, where $0<b \leq 1$;

(ii) $[a, b]$ or $(a, b]$ or $(a, b)$, where $-1 \leq a<0$ and $a^{2} \leq b \leq 1$;

(iii) $[a, b)$, where $-1<a<0$ and $a^{2} \leq b \leq 1$.

In [4] Kemprasit, Danpattanamongkon and Savettaseranee proved that no semigroup of type (i), (ii) or (iii) admits a ring structure. In this section we apply Theorem 1.3, and adopt some ideas of [4], to give an alternative and more consistent proof of the result.

We begin with the following general observation. Recall that if $S$ is a semigroup and $s \in S$, then $s$ is said to be a central element of $S$ if $s x=x s$ for any $x \in S$, and $s$ is called a left cancellative element of $S$ if $s x=s y$ implies $x=y$ for any $x, y \in S$.

Theorem 3.1 Let $S$ be a semigroup and let s be a central, cancellative element of $S$. Then $S$ is a ring semigroup if and only if $S$ is a ring semigroup.

Proof If $S$ is a ring semigroup, then there exists an addition $+\operatorname{such}$ that $(S,+, \cdot)$ is a ring. Since $s S$ is a right ideal of the ring, $s S$ is a ring semigroup.

Conversely, let $s S$ be a ring semigroup. Then there exists an addition + such that $(s S,+, \cdot)$ is a ring. We define an operation $\oplus$ on $S$ as follows. For any $x, y \in S$, $s x+s y \in s S$, and thus there exists $t \in S$ such that $s x+s y=s t$. Since $s$ is left cancellative, such a $t$ is unique, and we put $x \oplus y=t$. In other words, $x \oplus y$ is a unique element of $S$ such that

$$
s x+s y=s(x \oplus y) .
$$

It is clear that $x \oplus y=y \oplus x$ for any $x, y \in S$. Furthermore, for any $x, y, z \in S$ we have

$$
\begin{aligned}
s(x \oplus(y \oplus z)) & =s x+s(y \oplus z)=s x+(s y+s z) \\
& =(s x+s y)+s z=s(x \oplus y)+s z=s((x \oplus y) \oplus z),
\end{aligned}
$$

which implies that $x \oplus(y \oplus z)=(x \oplus y) \oplus z$, and thus the operation $\oplus$ is associative. Moreover, if $x \in S$, then $s(0 \oplus x)=s 0+s x=0+s x=s x$, and thus $0 \oplus x=x$, showing that 0 is a zero element of $\oplus$. Furthermore, if $x \in S$, then there exists $y \in S$ with $s x+s y=0$. Hence $s(x \oplus y)=s 0$, and $x \oplus y=0$ follows, proving that $(S, \oplus)$ is an abelian group.

To complete the proof, we consider any elements $x, y, z \in S$. Since $s$ is central,

$$
\begin{aligned}
s(x(y \oplus z)) & =x(s(y \oplus z))=x(s y+s z)=x s y+x s z=s(x y)+s(x z) \\
& =s(x y \oplus x z)
\end{aligned}
$$


and $x(y \oplus z)=x y \oplus x z$ follows. Furthermore

$$
s((x \oplus y) z)=(s(x \oplus y)) z=(s x+s y) z=s x z+s y z=s(x z \oplus y z),
$$

which shows that $(x \oplus y) z=x z \oplus y z$. Thus $(S, \oplus, \cdot)$ is a ring. Hence $S$ is a ring semigroup.

We are now in a position to prove that no multiplicative interval semigroup of type (i) admits a ring structure (cf. [4, Corollaries 1,2]).

Lemma 3.2 Let $b \in \mathbb{R}$ be such that $0<b \leq 1$. Then neither of the multiplicative interval semigroups $[0, b]$ and $[0, b)$ is a ring semigroup.

Proof It is easy to see that $[0,1]$ and $[0,1)$ are right-chain semigroups. Hence by Corollary 1.4 neither of these two semigroups is a ring semigroup. We are left with the case where $b<1$. Then $b \in[0,1)$, and since $[0, b]=b[0,1]$ and $[0, b)=b[0,1)$, Theorem 3.1 implies that neither $[0, b]$ nor $[0, b)$ is a ring semigroup.

Next we turn to multiplicative interval semigroups of the form $[a, b]$ (cf. [4, Corollary 3]).

Lemma 3.3 If $-1 \leq a<0$ and $a^{2} \leq b \leq 1$, then the multiplicative interval semigroup $[a, b]$ is not a ring semigroup.

Proof We first prove the special case where $b=1$. For that we apply Theorem 1.3 with $A=(a, 1)$. By this theorem, to prove that $S=[a, 1]$ is not a ring semigroup, it suffices to show that for any $x, y \in(a, 1)$ there exists $z \in J=\{s \in S: s S \neq S\}$ with $x S \subseteq z S$ and $y S \subseteq z S$. Note that $J=[a, 1)$ if $a \neq-1$, and $J=(a, 1)$ if $a=-1$; in particular $A \subseteq J$. We can assume that $x<y$. The case where $x=0$ or $y=0$ is clear. If $0<x<y$, then $x=y \frac{x}{y} \in y S$, and thus we can put $z=y$ in this case. If $x<y<0$, then $y=x \frac{y}{x} \in x S$, and we can set $z=x$. We are left with the case where $a<x<0<y<1$. Then $x a<1, \frac{x}{a}<1$ and $y<1$, and thus there exists $z \in S$ with $\max \left\{x a, \frac{x}{a}, y\right\}<z<1$ (in particular $z \in J$ ). Since $z a<x<x a<z$ and $z a<y a<$ $y<z$, we have $x S=[x, x a] \subseteq[z a, z]=z S$ and $y S=[y a, y] \subseteq[z a, z]=z S$, as desired.

Now we prove the general case. If $a \geq-b$, then $-1 \leq \frac{a}{b}<0$ and thus, as we have already proved, $\left[\frac{a}{b}, 1\right]$ is not a ring semigroup. Hence $[a, b]=b\left[\frac{a}{b}, 1\right]$ is not a ring semigroup by Theorem 3.1. If $a<-b$, then $-1<\frac{b}{a}<0$, and thus $\left[\frac{b}{a}, 1\right]$ is not a ring semigroup. Hence by Theorem 3.1, $a^{2}\left[\frac{b}{a}, 1\right]=\left[a b, a^{2}\right]=a[a, b]$ is not a ring semigroup, and thus by the same theorem, $[a, b]$ is not a ring semigroup.

Now we consider the remaining bounded interval semigroups on $\mathbb{R}$ (cf. [4, Corollary 4]). 


\section{Lemma 3.4}

(1) If $-1 \leq a<0$ and $a^{2} \leq b \leq 1$, then neither of the multiplicative interval semigroups $(a, b]$ and $(a, b)$ is a ring semigroup.

(2) If $-1<a<0$ and $a^{2} \leq b \leq 1$, then the multiplicative interval semigroup $[a, b$ ) is not a ring semigroup.

Proof Parts (1) and (2) follow by the same argument. Indeed, let $S$ be any of the semigroups $(a, b],(a, b)$ or $[a, b)$. For any $n \in \mathbb{N}$ set $d_{n}=\frac{b}{2}+\frac{b}{3 n}$. Then $d_{n} \in(a, b)$, and thus $d_{n} \in S$. Furthermore $\left[\frac{a b}{2}, \frac{b^{2}}{2}\right] \subseteq d_{n} S \subseteq\left[a d_{n}, b d_{n}\right]$. Since $\lim _{n \rightarrow \infty}\left(a d_{n}\right)=\frac{a b}{2}$ and $\lim _{n \rightarrow \infty}\left(b d_{n}\right)=\frac{b^{2}}{2}$, it follows that $\left[\frac{a b}{2}, \frac{b^{2}}{2}\right]=\bigcap_{n \in \mathbb{N}} d_{n} S$. Hence if $(S,+, \cdot)$ is a ring for an addition + , then $\left[\frac{a b}{2}, \frac{b^{2}}{2}\right]$ is an ideal of the ring, and thus $\left[\frac{a b}{2}, \frac{b^{2}}{2}\right]$ is a ring semigroup, contradicting Lemma 3.3.

As an immediate consequence of Lemmas 3.2, 3.3 and 3.4, we obtain the following result.

Theorem 3.5 [4] The trivial semigroups $\{0\}$ and $\{1\}$ are the only bounded multiplicative interval semigroups on $\mathbb{R}$ admitting a ring structure.

Acknowledgements The author was supported by MNiSW Grant Nr N N201 268435.

Open Access This article is distributed under the terms of the Creative Commons Attribution Noncommercial License which permits any noncommercial use, distribution, and reproduction in any medium, provided the original author(s) and source are credited.

\section{References}

1. Anderson, D.D., Stickles, J.: Commutative rings with finitely generated multiplicative semigroups. Semigroup Forum 60, 436-443 (2000)

2. Ferrero, M., Mazurek, R., Sant'Ana, A.: On right chain semigroups. J. Algebra 292, 574-584 (2005)

3. Hajnal, A., Kertész, A.: Some new algebraic equivalences of the Axiom of Choice. Publ. Math. (Debr.) 19, 339-340 (1972)

4. Kemprasit, Y., Danpattanamongkon, Ng., Savettaseranee, K.: Some results on semigroups admitting ring structure. ScienceAsia 36, 85-88 (2010)

5. Kogalovski, S.R.: On multiplicative semigroups of rings. Dokl. Akad. Nauk SSSR 140, 1005-1007 (1961) (in Russian)

6. Lawson, L.J.M.: The multiplicative semigroup of a ring. Doctoral dissertation, Univ. of Tennessee (1969)

7. Oman, G.: Ring semigroups whose subsemigroups form a chain. Semigroup Forum 78, 374-377 (2009)

8. Pearson, K.R.: Interval semirings on $R_{1}$ with ordinary multiplication. J. Aust. Math. Soc. 6, 273-288 (1966)

9. Peinado, R.E.: On semigroups admitting ring structure. Semigroup Forum 1, 189-208 (1970)

10. Satyanarayana, M.: On semigroups admitting ring structure. Semigroup Forum 3, 43-50 (1971) 